NEUROSCIENCE

\section{Tracing glutamate}

Nat. Meth. 10, 162-170 (2013)

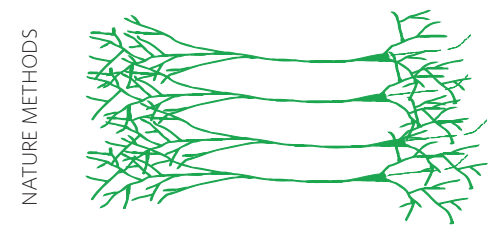

Glutamate receptors are critical for synaptic transmission and regulation of neuronal activity. Interrogating the direct determinants of neural circuit function thus requires sensors capable of monitoring rapid neurotransmitter transients in intact preparations. To overcome limitations of existing glutamate biosensors, Marvin et al. fused a bacterial periplasmic glutamatebinding protein to circularly permuted GFP such that the sensor responds to glutamate with green light. iGluSnFR is specific for glutamate over other amino acids, neurotransmitters or drugs and has a high signal-to-noise ratio. In cultured neurons and astrocytes, iGluSnFR responded to glutamate release from single electrical pulses. Glutamate uncaging in brain slices calibrated the sensor signal to physiological neurotransmission levels. In mouse retina in vitro, Caenorhabditis elegans worms, and zebrafish larvae and rat motor cortex in vivo, iGluSnFR reported glutamate transients in single neurons, dendritic spines and dendritic branches in awake, active animals, demonstrating its utility for monitoring excitatory synaptic transmission. iGluSnFR and sensors for other neurotransmitters could prove useful in combination with calcium imaging to fully document how neuronal signaling events integrate to brain activity. $M B$

\section{HETEROCYCLES}

\section{Stitching in selenium}

ChemBioChem, published online 18 February 2013; doi:10.1002/cbic.201300037

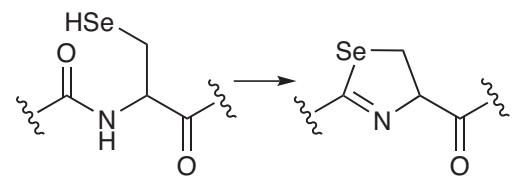

Selenium provides a structurally similar but chemically distinct counterpart to the oxygen and sulfur atoms commonly found in medicinal chemistry libraries, but the toxic precursors needed to synthesize selenium-containing heterocycles have limited its broader application. To facilitate access to complex Se-containing structures, Koehnke et al. report that two biosynthetic enzymes can cyclize selenium-containing peptides. PatD and TruD-heterocyclases from the patellamide and trunkamide pathways-normally catalyze the formation of five-membered rings in their peptide substrates by condensing a serine or cysteine side chain with a neighboring backbone carbonyl. The authors thus wondered whether the enzymes might also be able to tolerate selenocysteine (SeCys),

\section{PLURIPOTENCY}

Tet à tet
Nature, published online 10 February 2013; doi:10.1038/nature11925

Transcription factors such as NANOG are essential to reprogram somatic cells to a pluripotent state, but little is known about the protein-protein interaction networks that might be necessary for this important biological activity. Costa et al. apply an improved version of affinity purification-MS with a bioinformatics analysis of the resulting data to identify 27 protein-interaction partners for NANOG, including the methylcytosine hydroxylases TET1 and TET2. Interaction between the TET proteins and NANOG was confirmed by coimmunoprecipitation. Genetic knockdown of TET1 or TET2 decreased reprogramming efficiency in multiple reprogramming models. Conversely, ectopic expression of catalytically active forms of TET1 or TET2 improved reprogramming efficiency, indicating that TET enzymatic activity contributes to its impact on reprogramming. The authors then demonstrated that 5 -hydroxylmethylcytosine ( $5 \mathrm{hmC}$ ) levels were increased by the combined expression of NANOG and TET1; chromatin immunoprecipitation-sequencing data revealed that NANOG and TET1 overlap substantially on chromatin and are enriched at both pluripotency and developmental gene promoters. Indeed, localization of TET1 to these promoters was dependent upon the expression of NANOG and correlated with increased $5 \mathrm{hmC}$ at these loci. Taken together, these data indicate that NANOG recruits methylcytosine hydroxylase activity to loci involved in reprogramming and that the hydroxylation of $5 \mathrm{mC}$ at particular promoters has an important role in the reprogramming process.

the twenty-first amino acid, in their active sites. Supplementation of Escherichia coli cultures with SeCys led to incorporation of this residue in two related peptide sequences. The linear peptides could be derivatized with iodoacetamide, providing a convenient test for PatD- or TruD-mediated cyclization, which was confirmed via proteolytic digestions and MS. Surprisingly, formation of the mature selenazolines proceeded as quickly as the formation of thiazolines in the native sequences. This plasticity, combined with prior estimates that one quarter of bacteria have the genetic capacity to make SeCys, led the authors to speculate that naturally occurring selenium-containing compounds may await discovery. $C G$

TRANSCRIPTIONAL REGULATION

\section{Find that factor}

Proc. Natl. Acad. Sci. USA 110, 3645-3650 (2013)

The transcription of a gene can be negatively or positively regulated when a transcriptional repressor or activator, respectively, binds a specific DNA sequence, typically within the promoter region of the gene. Unraveling how a gene is regulated can be very challenging, as there may be many different transcription factor-binding sites near a single gene, with overlapping binding sites confounding the issue. Mirzaei et al. now report a method that uses MS to systematically monitor hundreds of putative transcriptional regulators to determine which ones actually bind a specific promoter sequence in Saccharomyces cerevisiae nuclear extracts. After showing that their methodology was reproducible, the authors used it to identify transcription factors that interacted with the FLO11 promoter, which contains at least four upstream activation sequences and nine repression elements. Of the 222 known or putative transcriptional regulators evaluated in this experiment, the method identified 15 proteins that bound at least one of the three segments of the FLO11 promoter that were assayed. Follow-up experiments confirmed that the authors had identified a transcriptional repressor (Mot3) and a transcriptional activator (Azf1) of FLO11 expression in S. cerevisiae. Comparison of their method with other systematic transcriptional regulator-DNA interaction methodologies-for example, chromatin immunoprecipitation-based approaches-indicates that the approaches are largely complementary, with some uniquely identified transcriptional regulators in each data set. 